\title{
Experimental investigation of the thermal properties of tailored expansion lattices
}

\author{
Craig A. Steeves $\cdot$ Chris Mercer • \\ Emilio Antinucci $\cdot$ Ming Y. He • \\ Anthony G. Evans
}

Received: 25 June 2008/Accepted: 12 February 2009/Published online: 17 March 2009

(C) The Author(s) 2009. This article is published with open access at Springerlink.com

\begin{abstract}
Composite bimaterial lattice structures which possess both low, tailorable thermal expansion and nearly optimal stiffness have been proposed for applications which require high structural stiffness in environments which include large temperature fluctuations, such as the surfaces of high-speed aerospace vehicles. An experimental validation of the thermal properties of these lattices when they are constructed of practical materials with easily manufactured bonded joints is contained herein. Bonded lattices, comprising aluminum and titanium alloys, have been manufactured with press-fit dovetail joints and tested in a variety of thermal environments. Results for equilibrium heating, rapid transient heating and thermal cycling leading to shakedown are presented and shown to be consistent with theoretically and numerically attained results.
\end{abstract}

Keywords Low thermal expansion . Composite structures · Experimental testing

C. A. Steeves $(\square) \cdot$ C. Mercer · E. Antinucci ·

M. Y. He - A. G. Evans

Materials Department, University of California, Santa

Barbara, Santa Barbara, CA 93106, USA

e-mail: csteeves@utias.utoronto.ca

C. Mercer

National Institute for Materials Science, 1-2-1 Sengen,

Tsukuba, Ibaraki 305-0047, Japan

\section{Introduction}

In structural systems that experience large temperature changes and thermal gradients, the associated strains are a significant impediment to successful design and implementation. The large thermal strains result in excessive geometric changes and substantial thermal stresses when high-temperature components are connected to lower temperature structures with smaller thermal strains. The consequences include failure by yielding, fracture or fatigue, as well as the formation of gaps that require sealing and extreme forces on attachments to other structures.

Multimaterial lattices have been proposed as a solution to these issues. By combining two or more materials with empty space, composite lattice structures can be manufactured to have a thermal expansion coefficient which is tailored to the application (and which can be zero or even negative). Lakes (1996), Sigmund and Torquato (1996), Gibiansky and Torquato (1997), and Jefferson (2006) have all proposed lattice structures with these properties. However, the Lakes and Jefferson lattices are both bending-dominated structures (see Deshpande et al. 2001) and are consequently very compliant, while the structures proposed by Torquato and his collaborators are biaxially (but not uniaxially) stiff but very difficult to manufacture. More recently, Grima et al. (2007) demonstrate a lattice material which is stiff but has highly anisotropic thermal properties. 
To overcome such limitations, a family of stiff, thermally isotropic tailored expansion lattices has been proposed by Steeves et al. (2007a). The lattice configuration (Fig. 1) is composed of two materials with widely differing individual coefficients of thermal expansion (CTEs) and intervening spaces. In this concept, the thermal expansions of the individual members upon heating are accommodated by rotation at the nodes, allowing for small net changes in lattice dimensions. That is, when heated, the higher CTE $\left(\alpha_{2}\right)$ internal triangular members expand more rapidly and push outward against the lower CTE $\left(\alpha_{1}\right)$ exterior lattice. This causes the latter to rotate, thereby counteracting the lengthwise elongation of the members. The two critical parameters are the lattice skewness, $\theta$ (the deviation from a perfect equilateral triangle of the lower CTE continuous lattice members) and the thermal expansion ratio, $\Sigma=\alpha_{2} / \alpha_{1}$, of the two constituent materials. The ensuing dependency for pin-jointed lattices, from the model described in Steeves et al. (2007a), is plotted on Fig. 2, wherein the net thermal expansion of the lattice $\bar{\alpha}$ is normalized by the thermal expansion of the lower CTE constituent, $\alpha_{1}$. Note that a wide range of lattice CTE is possible, including zero and even negative values, and that the lattice properties are scale-independent and can be implemented on the microscale given appropriate manufacturing techniques.

A key aspect of this lattice design is its high overall stiffness compared to other lattices with low thermal expansion. The lattice described here is

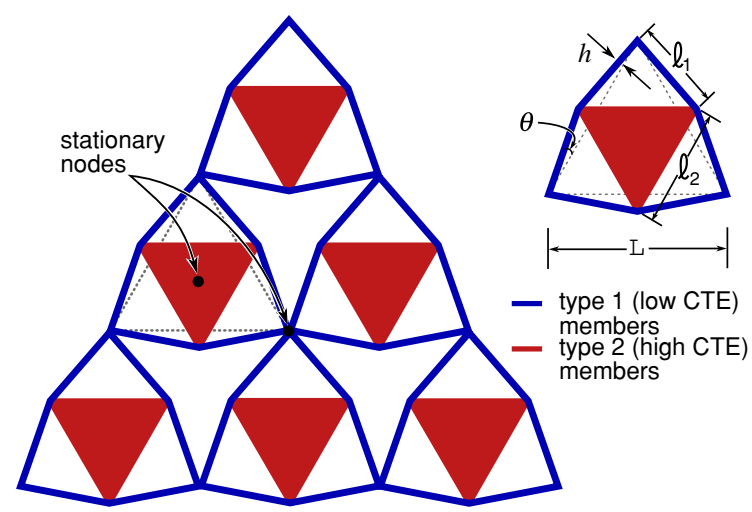

Fig. 1 A sketch of the lattice structure showing the key geometric parameters. The low CTE type 1 members are blue while the high CTE type 2 members are red. The key parameters are the lattice skewness $\theta$ and the ratio of thermal expansions $\Sigma=\alpha_{2} / \alpha_{1}$

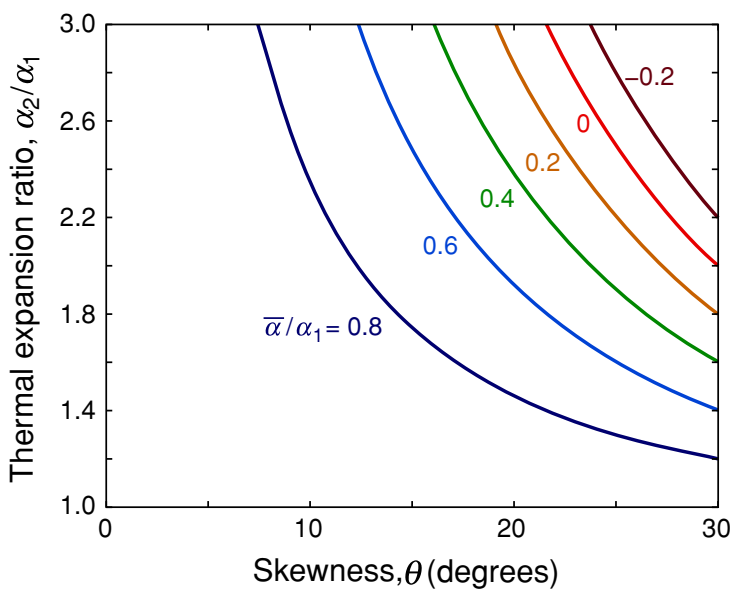

Fig. 2 Contours of the normalized net lattice coefficient of thermal expansion, plotted as functions of the lattice skewness $\theta$ and the thermal expansion ratio $\Sigma=\alpha_{2} / \alpha_{1}$

stretching-dominated (see Deshpande 2001), and hence is approximately one order of magnitude stiffer than comparable bending-dominated structures. Gibiansky and Torquato (1997) developed a theoretical bound for the biaxial stiffness for bimaterial lattices which have zero thermal expansion. The structural performance of this lattice lies close to the theoretical bound and hence the lattice is nearly optimally stiff; see Steeves et al. (2007a) for details.

These lattices can be constructed using many material options, including high-temperature refractory alloys. These form the basis for application of the concept to the hot exterior faces of a thermal protection panel, which combines bending stiffness with thermal resistance, while reducing overall mass (Steeves et al. 2007b). In such panels, the stationary nodes (Fig. 1), allow the lattice to be connected to cool vehicle structures without generating thermal stresses. The results in Fig. 2 have been derived for a pin-jointed lattice and verified experimentally for such a system. In practice, because of manufacturing limitations, the lattices are bonded at the nodes. Such bonding increases the effective lattice CTE from the ideal values for pinned systems. The focus of the present article is on the behavior of a system with bonded nodes.

The modifications when the nodes are bonded will be explored through a combination of beam theory analysis (Steeves et al. 2007a), finite element analysis and measurements conducted on a lattice with a $\mathrm{Ti}$ alloy as the lower CTE lattice $\left(\alpha_{1} \sim 9.5 \mathrm{ppm} /{ }^{\circ} \mathrm{C}\right)$ and 
an $\mathrm{Al}$ alloy as the higher CTE constituent $\left(\alpha_{2} \sim 24\right.$ $\left.\mathrm{ppm} /{ }^{\circ} \mathrm{C}\right)$. The nodes use a mechanical attachment scheme (rather than brazing or welding). The system was designed using the beam theory solution for the bonded lattice to have an overall CTE about half that for the lower CTE constituent: $\bar{\alpha} \sim 4 \mathrm{ppm} /{ }^{\circ} \mathrm{C}$. This choice was made because, in applications such as the thermal protection panel, the thermal strains of the hot and cool faces must be matched (that is, zero CTE is not desirable). An image of the lattice (Fig. 3a) illustrates the configuration.

To elucidate the lattice behaviour, the paper is organized as follows. The materials used and the lattice fabrication are described, followed by a description of the measurement procedures. As a guide for the measurements, the thermal expansion analysis is presented for equilibrium heating and cooling. This is followed by the measurements for equilibrium and transient heating, as well as the local behavior at the joints, with a particular focus on shakedown during thermal cycling. Finally, some summary comments will be provided.

\section{Materials, fabrication and experimental methods}

The lattice comprises $3 \times 3$ unit cells incorporating lower CTE Ti-6Al-4V and higher CTE Al 7075-T6 (Fig. 3a), cut by electric discharge machining from $3 \mathrm{~mm}$ thick plate. The unit cells are of length $L=50 \mathrm{~mm}$, with strut width $h=3 \mathrm{~mm}$ for the type 1 members. The type 2 components were solid triangles. The assembly employs a press-fit dovetail joint with an interference of $20 \mu \mathrm{m}$ (Fig. 3b). Properties of the constituent materials are presented in Table 1. The dimensions and characteristic geometric parameters are provided in Table 2.

For precise monitoring of thermal displacements upon heating and cooling, a black and white speckle pattern was applied to one face of the lattice with high-temperature paint. Pre-test digital, optical images were taken with the aid of a high resolution camera (AVT Dolphin F-201B, 8-bit black/white $1628 \times 1236$ progressive $\mathrm{CCD}$ array) and used as a reference. The lattices were heated by placing them

Fig. 3 a The $3 \times 3$ lattice comprising lower CTE Ti-6Al-4V and higher CTE Al-7075-T6, assembled with b press-fit dovetail joints
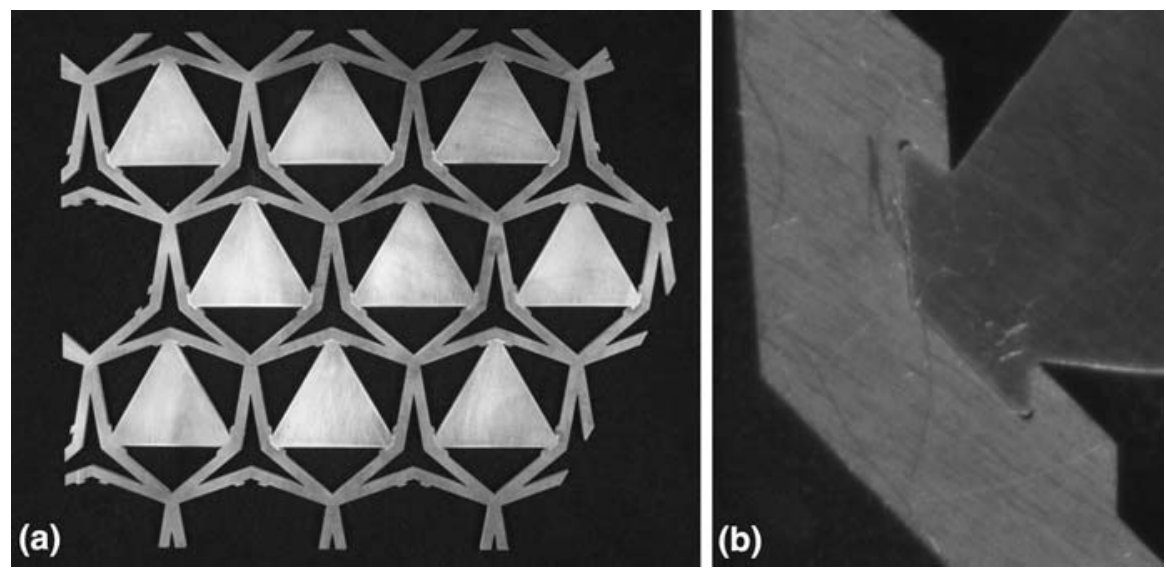

Table 1 Material properties for the titanium and aluminium alloys used in the numerical simulations, including the variation of yield strength and thermal expansion with temperature

\begin{tabular}{|c|c|c|c|c|c|}
\hline \multirow[t]{2}{*}{ Material } & \multirow{2}{*}{$\begin{array}{l}\text { Young's } \\
\text { Modulus (GPa) }\end{array}$} & \multicolumn{2}{|c|}{ Yield Strength } & \multicolumn{2}{|c|}{ Thermal Expansion } \\
\hline & & $\sigma_{Y}(\mathrm{MPa})$ & $\mathrm{T}\left({ }^{\circ} \mathrm{C}\right)$ & $\alpha\left(\mathrm{ppm} /{ }^{\circ} \mathrm{C}\right)$ & $\mathrm{T}\left({ }^{\circ} \mathrm{C}\right)$ \\
\hline \multirow[t]{3}{*}{ Al alloy 7075-T6 } & & 434 & 20 & 22.9 & 20 \\
\hline & 70 & 391 & 200 & & \\
\hline & & 339 & 300 & 26.0 & 300 \\
\hline \multirow[t]{2}{*}{ Ti alloy Ti-6Al-4V } & 110 & 1100 & 20 & 9.2 & 20 \\
\hline & & 858 & 300 & 9.6 & 300 \\
\hline
\end{tabular}


Table 2 The geometric parameters of the lattice shown in Fig. 3

\begin{tabular}{lllll}
\hline $\begin{array}{l}\text { Skewness } \\
\theta \text { (degrees) }\end{array}$ & $\begin{array}{l}\text { Unit cell } \\
\text { length } \\
L(\mathrm{~mm})\end{array}$ & $\begin{array}{l}\text { Type 1 } \\
\text { length } \\
\ell_{1}(\mathrm{~mm})\end{array}$ & $\begin{array}{l}\text { Type 2 } \\
\text { length } \\
\ell_{2}(\mathrm{~mm})\end{array}$ & $\begin{array}{l}\text { Strut } \\
\text { width } \\
h(\mathrm{~mm})\end{array}$ \\
\hline 20 & 49.6 & 25.5 & 35.4 & 3 \\
\hline
\end{tabular}

onto a hot plate incorporating a heat-pipe that enables uniform and steady heating across the entire surface. A glass cover plate prevented convection currents that lead to heat shimmer and optical fluctuations. The expansion of the hot plate, and the consequent relative approach of the specimen to the camera, was monitored to remove this effect from the calculation of CTE. To ascertain thermally-induced displacements, images were taken at regular temperature intervals (typically $10^{\circ} \mathrm{C}$ ), up to a final temperature of $220^{\circ} \mathrm{C}$, and analyzed using digital image correlation software (Correlated Solutions 2006). The software is capable of detecting displacements of the speckle pattern with a resolution of 0.01 pixels, translating in a spatial resolution of $0.31 \mu \mathrm{m}$ using a $50 \mathrm{~mm}$ field of view. Calibration measurements at ambient temperature indicated an accuracy within $2 \%$.

The measurements were taken at two lattice points located on the middle cell; that is, the only cell in the $3 \times 3$ lattice entirely surrounded by other cells. Temperatures were recorded using four digital read- expansion measurement was then repeated. With the same lattice, this process was repeated for 60 total cycles. Localized strains occurring in the vicinity of the joints were obtained on unit cells of the same lattice configuration using a $15 \times 15$ pixel sampling region, in conjunction with close-up photographs taken before and after thermal cycling.

The influence of heating transients on the displacements has also been examined. To achieve abrupt heating, the hot plate was preheated to $250^{\circ} \mathrm{C}$ and the lattice, at ambient temperature, placed in contact with the hot surface. During the tests a diminishing difference in the temperature of the two constituents was observed, eventually vanishing (less than $3^{\circ} \mathrm{C}$ ) after approximately $2 \mathrm{~min}$.

\section{Thermal expansion analysis}

The theory of pin-jointed and bonded lattices has been fully elucidated in Steeves et al. (2007a). To summarize, for a pinned lattice in equilibrium heating conditions, the net thermal expansion is given by:

$\frac{\bar{\alpha}}{\alpha_{1}}=\frac{1-\frac{1 \alpha_{2}}{2 \alpha_{1}} \sin (2 \theta)\left(\frac{1}{\sqrt{3}}+\tan \theta\right)}{1-\frac{1}{2} \sin (2 \theta)\left(\frac{1}{\sqrt{3}}+\tan \theta\right)}$,

which is plotted in Fig. 2, as is the corresponding expression for bonded joints:

$$
\frac{\bar{\alpha}}{\alpha_{1}}=1-\frac{\left(C_{1} \tan \theta-12 \sqrt{3}\right)(\cos \theta+\sqrt{3} \sin \theta)\left(\frac{\alpha_{2}}{\alpha_{1}}-1\right)}{C_{1}(\sqrt{3} \cos \theta-\sin \theta)+12\left(\sqrt{3}+2 \frac{E_{1} A_{1}}{E_{2} A_{2}}\right)(\cos \theta+\sqrt{3} \sin \theta)}
$$

out Chromel-Alumel thermocouples, two each positioned on the titanium and aluminum members. The lattice temperature was taken to be the average of the four thermocouples.

To examine the stability of the thermal expansion, as well as the role of shakedown at the joints, after the first test, the lattice was placed in an electric furnace and subjected to 28 thermal cycles, each comprising $30 \mathrm{~min}$ at $250^{\circ} \mathrm{C}$ followed by cooling to ambient temperature for $20 \mathrm{~min}$. The thermal with $E_{1}$ and $E_{2}$ the Young's moduli and $A_{1}$ and $A_{2}$ the cross-sectional areas of the two constituents. The parameter $C_{1}=A_{1} \ell_{1}^{2} / I_{1}$, where $I_{1}$ is the second moment of area of the lower CTE members. ${ }^{1}$ The predicted thermal expansion of the lattice is presented in Fig. 4, using the material properties from Table 1 and the dimensions from Table 2.

\footnotetext{
1 The exponent in the square was omitted from this equation in Steeves et al. (2007a).
} 


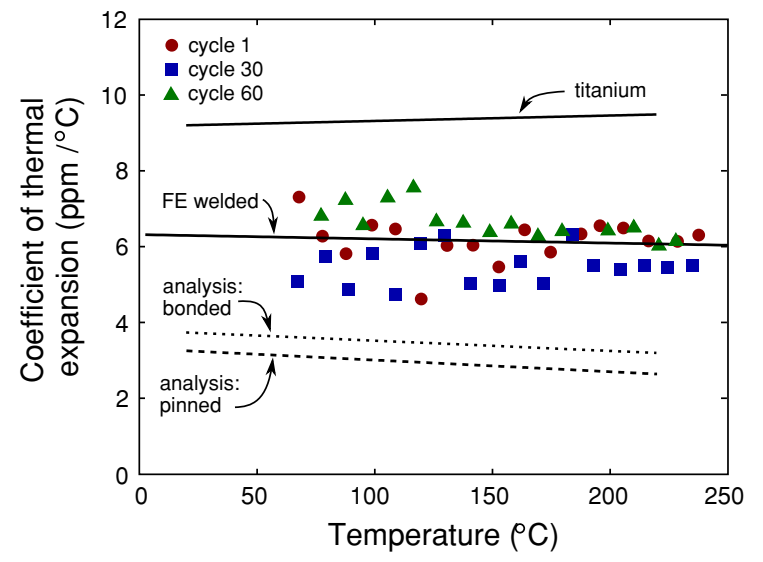

Fig. 4 Thermal expansion as a function of temperature for the bonded lattice. The beam theory prediction is indicated. Measurements conducted in the as-manufactured state as well as after 30 and 60 thermal cycles are superposed. The predicted results for a pinned lattice and the measured properties of the $\mathrm{Ti}$ alloy are included for comparison, as are the finite element predictions for this geometry and material combination with welded joints

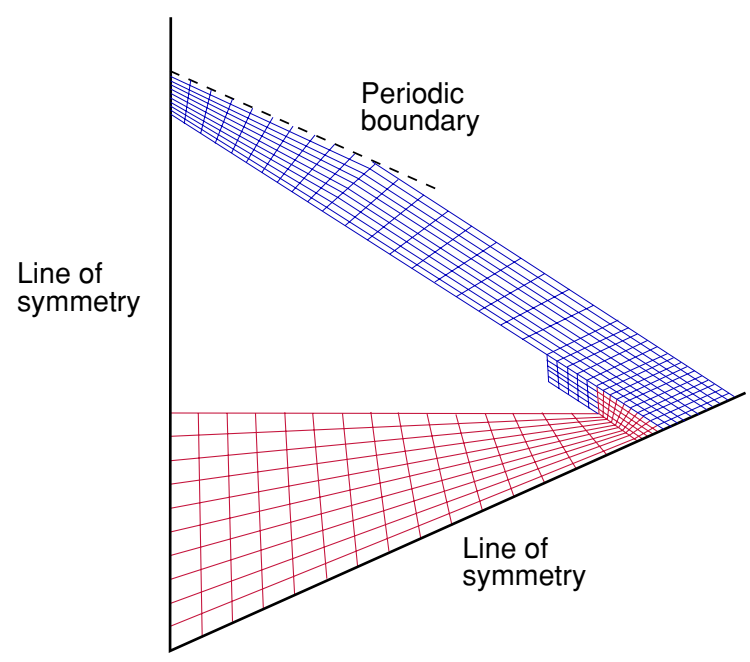

Fig. 5 Mesh scheme and symmetry used in the simulations. The lower CTE Ti-6V-4Al is in blue, while the higher CTE Al7075-T6 is in red. Because of symmetry, only one sixth of the unit cell need be modeled

Finite element simulations have been performed using the commercial package ABAQUS (HKS 2005). The same geometry as the experimental specimens was used with approximately 600 8-node biquadratic plane stress elements (Fig. 5). Using the symmetries of the system, only one sixth of the cell was modeled, with periodic boundary conditions. The

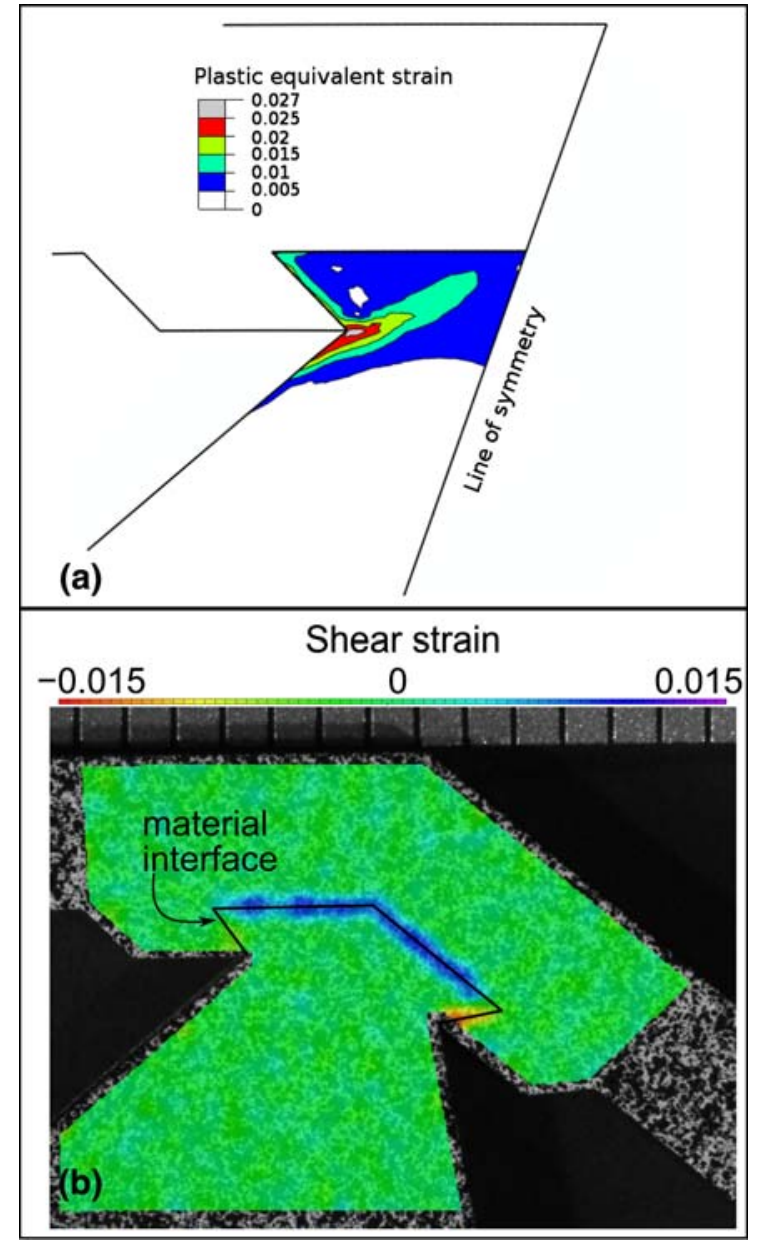

Fig. 6 Interface strains at the bonded joint after 30 cycles between room temperature and $250^{\circ} \mathrm{C}$. (a) Finite element predictions, and (b) measurements

temperature dependent properties of the two alloys are used (Table 1).

Two different conditions at the joint interface were simulated: one with Coulomb friction $(\mu=1)$ and the other with no slip. Multiple temperature cycles were used to ascertain the stability of the CTE and the incidence of local plastic strains at the joint. For both interface conditions, highly localized plastic strains accumulated during the first few thermal cycles (Fig. 6a). The strain at the interface reaches a maximum of $2.7 \%$ at the corner of the aluminum; along the length of the interface the strain is typically between 1.5 and $2.0 \%$. The associated changes in the overall lattice expansion are revealed on Fig. 7. Plastic strain at the attachment occurs during the first 


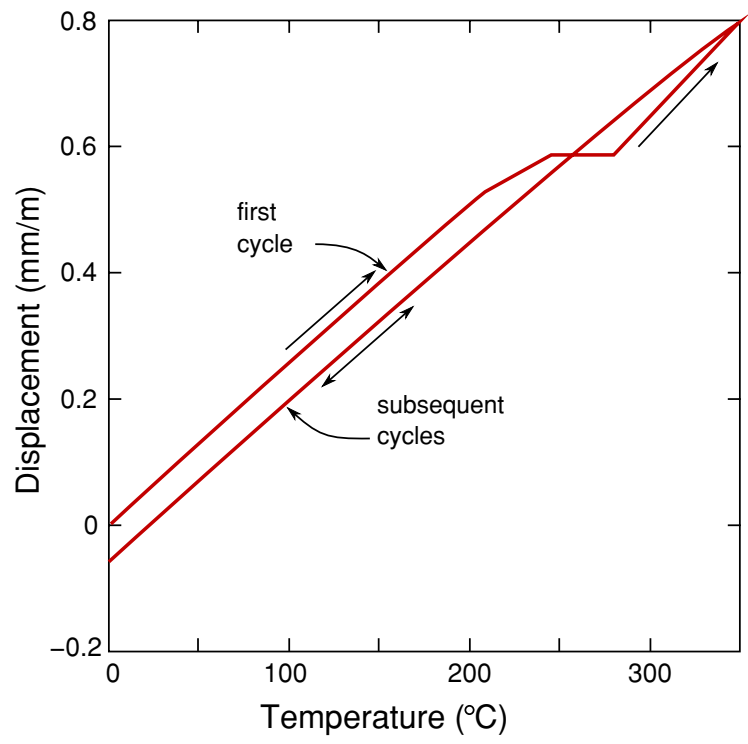

Fig. 7 Displacements calculated by finite elements of the lattice during the first and subsequent heating cycles, after which the lattice shakes down. Subsequent thermal cycles exhibit displacements which are repeatable

heating cycle, at $\sim 200^{\circ} \mathrm{C}$. Above this temperature there is a region where the temperature increases without an accompanying change in the lattice length. Thereafter, in all subsequent cycles, the expansion and contraction of the lattice is linear with no hysteresis, affirming that shakedown has occurred: the ensuing CTE is essentially unaffected by the interface deformations. In other locations on the lattice, including at the joints between the type 1 members, the stresses predicted by the finite element simulations are under all conditions insufficient to cause yielding.

\section{Experimental measurements}

\subsection{Equilibrium heating}

A representative strain contour image used to evaluate the thermal displacements is presented on Fig. 8. The equilibrium values of the thermal expansion coefficient measured using images of this type, as a function of temperature, are superimposed on Fig. 4.

Measurements are shown in Fig. 4 for tests at 1, 30 and 60 thermal cycles. Only the results at temperatures above $50^{\circ} \mathrm{C}$ are included because of the large measurement uncertainties at the low temperatures. Measurements for the isotropic expansion of Ti 6Al$4 \mathrm{~V}$ are also plotted. In all cases, the lattice CTE is approximately constant. This regularity with cycling is consistent with the finite element result indicating that shakedown occurs within the first few cycles.

The analytical predictions for bonded joints are significantly lower than the measurements: approximately $4 \mathrm{ppm} /{ }^{\circ} \mathrm{C}$ for the prediction versus $6 \mathrm{ppm} /{ }^{\circ} \mathrm{C}$ for the measurement. The discrepancy is because of the geometry of the joints in the titanium lattice, which are less compliant than ideal joints in slender beams, causing the joint rotations to be smaller than predicted. Similarly, the pinned configuration has greater compliance at the joints than the bonded geometry, further decreasing the net thermal expansion. This indicates that care must be exercised in using Eqs. 1 and 2 in design; increasing the compliance of the joints will ensure that the manufactured lattices more closely approach the analysis. Finite element simulations for an identical lattice with bonded joints which are welded (permitting no
Fig. 8 Strain contour plot of the sample at $180^{\circ} \mathrm{C}$, obtained through image analysis
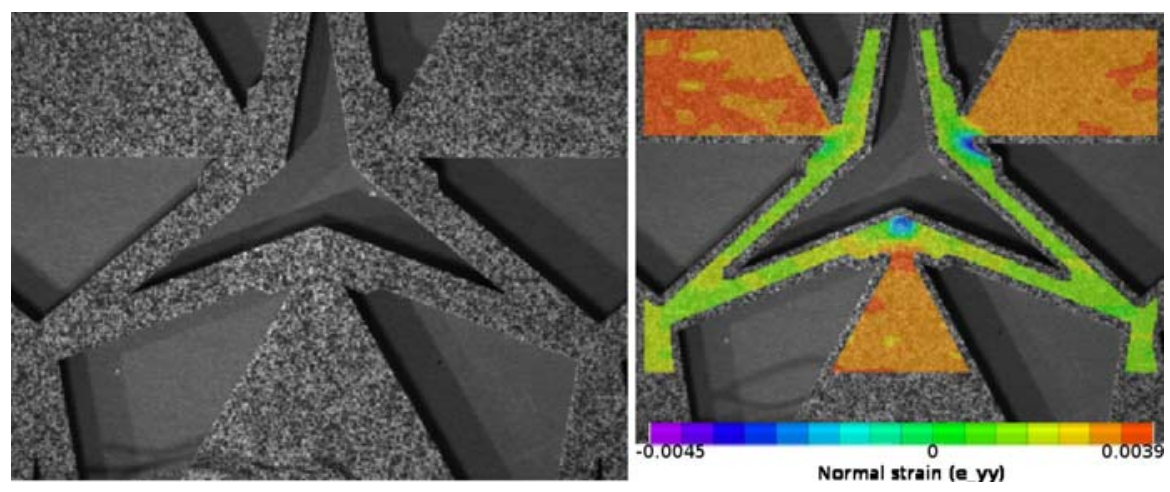
frictional slippage) are superimposed on Fig. 4; they match the experimental results well.

\subsection{Transient heating}

When the lattice is suddenly exposed to a heat source, the $\mathrm{Al}$ alloy members are initially hotter than their $\mathrm{Ti}$ counterparts because of the considerable difference in the thermal conductivities of the two alloys $(130 \mathrm{~W} /$ $\mathrm{mK}$ for $\mathrm{Al}$ and $6.7 \mathrm{~W} / \mathrm{mK}$ for $\mathrm{Ti}$ ). As the overall temperature increases, the difference between the two constituents decreases. During the transient, while a temperature difference exists, the thermal expansion of the lattice deviates from that anticipated by Fig. 8 . To characterize the transient, the normalized displacement of the lattice nodes has been selected as the measurement metric. Results are plotted against temperature in Fig. 9a and compared with the corresponding measurements for equilibrium heating after 30 cycles. The temperatures depicted by the continuous line are the average for the two constituents. Those shown as circles and diamonds are for the $\mathrm{Ti}$ and $\mathrm{Al}$ constituents, respectively. The transient lattice displacements are smaller than those for equilibrium heating. This reduction is most marked at lower temperatures and vanishes around $200^{\circ} \mathrm{C}$, where the displacements coincide. The displacement deviations at the lower temperatures arise because of the temperature difference between the constituents. (Note the proportionality between the temperature difference of the constituents and the displacements). Namely, a difference in temperature between the two lattice constituents gives rise to an effective CTE, which varies with temperature and differs from that for slow, equilibrium heating.

An effective CTE is calculated by using the average displacement in conjunction with the temperature change from ambient, as plotted on Fig. 9b. Clearly, during the initial transient period this measure results in a lower CTE than in the equilibrium condition, with the difference diminishing as the temperature increases. The explanation is as follows: early in the heating cycle the hotter Al members expand more rapidly than at equilibrium, interpreted as a temporarily higher effective CTE, and hence a larger ratio $\alpha_{2} / \alpha_{1}$. Referring to Fig. 2, this larger ratio leads to a lower net CTE for the same skewness, and hence the normalized displacement of the lattice is smaller. As the temperatures of the $\mathrm{Al}$ and $\mathrm{Ti}$

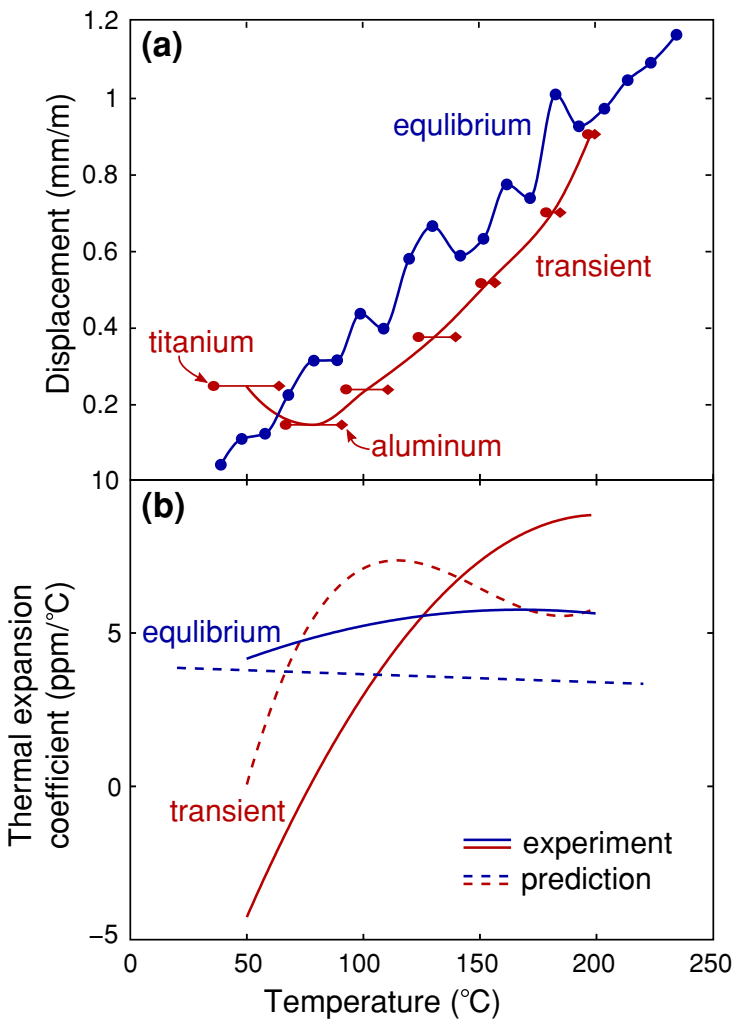

Fig. 9 Lattice transient behaviour after 30 thermal cycles. a Normalized extension of the lattice cell in equilibrium and during transient heating. The individual temperatures of the aluminum and titanium components are depicted as circles and diamonds respectively. b The effective thermal expansion coefficients during equilibrium and transient heating, displaying both experimental results (solid lines) and predictions (dashed lines)

equalise, the effective CTE increases to a value greater than for the equilibrium case, as the $\mathrm{Ti}$ is then heating more rapidly then the $\mathrm{Al}$ and the opposite reasoning holds. The measured temperatures and Eq. 2 predict transient behavior which is represented by a cubic curve fit in Fig. 9b and which exhibits the same overall trends as the measured behavior. The equivalent treatment for the equilibrium case is displayed for comparison.

\subsection{Local deformation and shakedown}

Figure $6 b$ is a contour plot of the residual shear strains after a unit cell is exposed to 30 thermal cycles and cooled to ambient. The interface is drawn onto the photograph for illustrative purposes. It is clear that the plastic strains are highly localized near the 
interface, due to the thermal expansion misfit at the contact, with a maximum shear strain of approximately $1.5 \%$. Moreover, after 30 further (60 total) thermal cycles (not shown), the plastic strains remain unchanged, affirming that shakedown has occurred. The strain pattern, as well as the strain levels, is comparable to those predicted by the FE calculations (Fig. 6a). After both 30 and 60 cycles, the lattice cells were manipulated manually and found to be structurally sound under the application of substantial forces; given that the cells were assembled by hand it is concluded that this level of residual strain is insufficient to compromise the structural integrity.

\section{Concluding comments}

Low CTE lattices comprising Ti-6Al-4V and $\mathrm{Al}$ 7075-T6 were manufactured and tested in several thermal environments: equilibrium heating, transient heating, and thermal cycling. When heated in equilibrium, the lattice behaviour was consistent with theoretical predictions with approximately $40 \%$ increase in CTE due to the non-ideal geometry of the lattice joints. When rapidly heated, the inequality of the thermal conductivities of the two constituents led to temperature differences between the aluminum and titanium. The consequence of this difference is an effective CTE which, for this combination of materials, was initially lower than the CTE in equilibrium heating during the rapid temperature increase of the higher CTE constituent, then higher than the equilibrium CTE as the temperatures of the constituents equalised. Unit cells which were thermally cycled to 30 and 60 cycles showed residual plastic strains at the interface between the constituents which were, in magnitude, comparable to finite element results. These plastic strains were not sufficient to compromise the structural integrity of the sample. Combined with finite element calculations which show shakedown after one cycle, this is strong evidence that thermal cycling does not affect the lattice structure, for this material combination and geometry.

Acknowledgments The authors would like to thank Peter Maxwell and Kirk Fields of UCSB for their assistance with the manufacture of specimens and speckle strain inferometry, respectively. The research reported in this paper was supported by the Office of Naval Research through a MURI program on Revolutionary Materials for Hypersonic Flight (Contract No. N00014-05-1-0439).

Open Access This article is distributed under the terms of the Creative Commons Attribution Noncommercial License which permits any noncommercial use, distribution, and reproduction in any medium, provided the original author(s) and source are credited.

\section{References}

Correlated solutions. Vic-2D users' manual, 4.4 edn. West Columbia, SC (2006)

Deshpande, V.S., Ashby, M.F., Fleck, N.A.: Foam topology: Bending versus stretching dominated architectures. Acta Mater. 9(6), 1035-1040 (2001)

Gibiansky, L.V., Torquato, S.: Thermal expansion of isotropic multiphase composites and polycrystals. J. Mech. Phys. Solids 45(7), 1223-1252 (1997)

Grima, J.N., Farrugia, P.S., Gatt, R., Zammitt, V.: A system with adjustable positive or negative thermal expansion. Proc. R. Soc. A 463(2082), 1585-1596 (2007)

HKS. ABAQUS/standard user's manual, 6.5 edn. Providence, RI (2005)

Jefferson, G.: Private communication. Air Force Research Lab (2006)

Lakes, R.S.: Cellular solid structures with unbounded thermal expansion. J. Mater. Sci. Lett. 15(6), 475-477 (1996)

Sigmund, O., Torquato, S.: Composites with extremal thermal expansion coefficients. Appl. Phys. Lett. 69(21), 32033205 (1996)

Steeves, C.A., Lucato, S.L., He, M.Y., Antinucci, E., Hutchinson, J.W., Evans, A.G.: Concepts for structurally robust materials that combine low thermal expansion with high stiffness. J. Mech. Phys. Solids 55(9), 1803-1822 (2007a)

Steeves, C.A., He, M.Y., Maxwell, P.T., Evans, A.G.: Design of a robust, multifunctional thermal protection system incorporating zero expansion lattices. In: Proceedings of the ASME International Mechanical Engineering Congress and Exposition. Seattle, Washington (2007b) 